\title{
Effects of Fast Hawk-Dove-Bully Game on the Dynamics of a Stage-structured Population
}

\author{
A. Moussaoui ${ }^{1}$, P. Auger ${ }^{2,3,4 *}$ \\ ${ }^{1}$ Department of Mathematics, Faculty of Sciences, University of Tlemcen. Algeria \\ 2 IRD, UMI 209, UMMISCO, IRD France Nord, F-93143, Bondy, France \\ ${ }^{3}$ UPMC, University Paris 6, UMI 209, UMMISCO, F-75005, Paris, France \\ ${ }^{4}$ UMMISCO-Dakar, University Cheikh Anta Diop, Dakar, Senegal
}

\begin{abstract}
In this paper, we present a review about mathematical models of population and community dynamics incorporating individual behaviour. The considered models consist in coupling a game theoretic model, going on at a fast time scale and that describes the change of tactics among individuals in a population or in a community to a model going on at a slow time scale describing the effects in the long term of individual tactics on reproduction and mortality of the populations. As application, an extension of a stage-structured population with intraindividual behavioural variability is presented. Individuals can adopt three different tactics: hawk, dove and bully. We consider a stage-structured population with immature and mature individuals. A mature individual experiences the different tactics in his life and it is assumed that the game is played at a fast time scale in comparison to population dynamics. This fast part of the model is coupled to a slow part, which describes the growth of each sub-population. By use of aggregation of variables methods, we obtain a reduced system of two differential equations governing immature and total mature sub-populations at the slow time scale. The analysis of the model shows that when the cost of fightings is smaller, the proportion of hawks is larger. The total population size equilibrium shows a maximum with respect to the gain of the game.
\end{abstract}

Keywords and phrases: game theory, Hawk-Dove-Bully model, population dynamics, aggregation of variables

Mathematics Subject Classification: 91A80, 92D25, 37N25

\section{Introduction}

Several authors consider that mathematical models have some limitations because they cannot take into account all the complexity of individual behaviour and their interactions. Therefore, a large class of Individual Based Models (IBM) have been developed. In these models, individuals usually move in a continuous space or in a network of patches. Individuals are assumed to achieve some tasks. When an individual comes close to another one, it interacts with its neighbour. As an example, the individual can modify its trajectory, its speed, its direction. In case of a predator coming close to a prey, it can attack the prey and may capture it with certain probability of success etc. Those IBMs intend to

\footnotetext{
${ }^{*}$ Corresponding author. E-mail: moussaoui.ali@gmail.com, pierre.auger@ird.fr
} 
take into account the complexity of individual interactions much better than classical mathematical models. However, in general, it is not possible to analyze the IBM model such as mathematical models. It is usually difficult to obtain general results such as existence of equilibria and local/global stability analysis, existence of periodic solutions, or else existence of attractors. As a consequence, many numerical simulations of the IBM starting at different initial conditions must be performed. In the next step, one expects to observe the emergence at the population/community level of some global behaviour. As an example, we refer to predator-prey models where an IBM was used in order to observe the emergence of functional responses in a predator-prey IBM incorporating complex individual behaviour of prey and predator [1]. We also refer to IBM looking for the emergence of group motion $[23,30]$.

There was a lot of interest in the study of interactions between individual behaviour and population dynamics $[20,39,40]$. At the level of population and community, instead of using IBMs, another approach consists in using population dynamics models in which individual behaviour is taken into account explicitely. For instance, we refer to spatial population models in a heterogeneous environment with different patches connected by migration with density-dependent immigration and emigration rates $[3-7,16,19,34,35]$. Therefore, those models take into account the decision to change patch for an individual depending on the presence/absence and density level of its own population and/or another population, which could be a competitor, a prey or a predator. We also mention models where the dynamic energy budget (DEB theory) of each individual is considered [24]. In a certain sense, these models are rather classical equation models but incorporate individual behaviour by considering different individual processes and mechanisms in the population dynamics. This leads to a certain complexity in the mathematical models and consequently to supplementary difficulties in the analysis of the models and the possibility to get analytical results.

On another hand, game models usually use the replicator equations, which are devoted to describe the variations of proportions of individuals playing a tactic among a population. However, the replicator equations do not take into account the variation of the total size of the populations. To our knowledge, few works considered game models taking into account population density variation. We nevertheless mention the work by Hauert et al. [21]. Our knowledge, few attention has been paid to mathematical models describing at the same time individual behaviour using a game theoretic approach and population-community dynamics using classical mathematical models.

In the present work, we focus on an approach using two different types of mathematical equations models: a mathematical model describing processes at the individual level (game model) and a mathematical model describing mechanisms at the population/community level. The complete model is finally obtained by coupling them together. For instance, one may consider a mathematical model that describes the interactions between individuals, using different tactics associated with different individual behaviours typically the replicator equations. One may also choose another model that describes the population and community dynamics, for instance a predator-prey model. The next step is to couple them to obtain a complete model. The individual model describes the interactions between individuals that may use different individual tactics. This model does not consider the variations of the population sizes but only of proportions of individuals using different individual tactics. The population-community model describes the variation of the population sizes due to mortality, birth, predation etc. This approach puts together the two different types of models, the game individual model and the population/community model to build a kind of meta-model. In this approach, it is considered that the complexity of individual interactions must be taken into account into a specific game model describing individual interactions among the population. The description of population dynamics needs another specific population-community model. The final step is to connect the two types of models by coupling them to give birth to a complete individual/population-community model. At our knowledge, only few works have been devoted to this general approach. The present paper is particularly aimed 
at presenting a review of this last class of complete individual/population-community models in section 2.

The paper is then organized as follows. In Section 3, we extend the original model in [32] to three tactics. It is structured into three subsections. Subsection 3.1 presents the hawk-dove-bully submodel at the fast time scale. Subsection 3.2 shows the stage structured model at the slow time scale. Subsection 3.3 presents the complete slow-fast model. Section 4 is devoted to present derivation of the aggregated model and our results regarding stability analysis of equilibria of this model and some numerical simulations. Section 5 presents a discussion of the results.

\section{A review of fast game models coupled to slow demographic models}

To start, let us give a short review of predator-prey models taking into account individual tactics used by predators to dispute a prey (see Auger et al. [12]). In this cited work, the authors proposed a preypredator model in which predators have two different individual behaviours: hawk and dove. A hawk predator is aggressive and always fights for the prey, while a dove predator never fights for the prey in the sense that it displays in any conflict and if it is attacked it immediately withdraws before it gets injured. Predators are split into two categories: hawk predators and dove predators, their densities are denoted by $p_{H}$ and $p_{D}$, respectively, let $n$ and $p$ be respectively the densities of preys and predators. The evolution of two populations is considered at two time scales: a fast time scale $\tau$ and a slow time scale $t$ such that $t=\varepsilon \tau$ where $\varepsilon$ is a small positive parameter that takes into account the two different time scales. The fast time scale is used to describe changes in tactics by predators, whereas the slow time scale is used to describe the birth, death and ecological interactions of predators and preys. The payoff matrix for hawk predators and dove predators is given by

$$
\mathbf{A}=\left(\begin{array}{cc}
\frac{G-C}{2} & G \\
0 & \frac{G}{2}
\end{array}\right)
$$

where $G$ is the gain of the game which will be assumed to be equal to the predation rate, i.e. the gain of a predator individual in the absence of fight with other predators, $C$ is the cost of the fight for prey between hawk predators, and $1 / 2$ presents the probability that a hawk predator gets the gain and cost in any conflict with the other hawk predator, and that a dove predator gets the gain in any encounter with another dove predator. At time $t$, the gain $\Delta_{H}$ of a predator always using the hawk strategy against a population with a proportion $x$ of hawk predators and $y$ of dove predators is the following one:

$$
\Delta_{H}=\left(\begin{array}{ll}
1 & 0
\end{array}\right) \mathbf{A}\left(\begin{array}{ll}
x & y
\end{array}\right)^{T} .
$$

The gain $\Delta_{D}$ of a predator always playing the dove strategy is the following one:

$$
\Delta_{D}=\left(\begin{array}{ll}
0 & 1
\end{array}\right) \mathbf{A}\left(\begin{array}{ll}
x & y
\end{array}\right)^{T} .
$$

The average gain of an individual playing the two tactics in proportions $(x(t), y(t))$ corresponding to the actual distribution of hawks and doves in the total population of predators is the following one:

$$
\Delta=(x y) \mathbf{A}(x y)^{T} .
$$

With these assumptions, the replicator equations [22] read

$$
\left\{\begin{array}{l}
\frac{d x}{d \tau}=x\left(\Delta_{H}-\Delta\right) \\
\frac{d y}{d \tau}=y\left(\Delta_{D}-\Delta\right)
\end{array}\right.
$$

In these equations, known as the replicator equations, it is assumed that when the gain of a strategy is higher than the average gain of the population, then the proportion of individuals playing this strategy 
increases with time (for more information about replicator equations and game dynamics, see [22,38]). On the slow time scale, it is assumed that the prey and predator obey the following system:

$$
\left\{\begin{array}{l}
\frac{d n}{d t}=r n\left(1-\frac{n}{K}\right)-a n p \\
\frac{d p_{H}}{d t}=p_{H}\left(\alpha \Delta_{H}-\mu\right) \\
\frac{d p_{D}}{d t}=p_{D}\left(\alpha \Delta_{D}-\mu\right)
\end{array}\right.
$$

Where $r$ is the growth rate of prey, $K$ is the carrying capacity, $\alpha$ is a conversion positive coefficient of gain and cost into biomass of predators and $\mu$ is the natural mortality rate assumed to be identical for hawks and doves. It is also assumed that the gain depends on the prey density as follows: $G(n)=a n$, where $a$ is a positive parameter.

By combining the two submodels on the fast time scale and slow time scale, we obtain the complete slow-fast predator-prey model

$$
\left\{\begin{array}{l}
\frac{d n}{d \tau}=\varepsilon\left(r n\left(1-\frac{n}{K}\right)-a n p\right) \\
\frac{d p_{H}}{d \tau}=p x\left(\Delta_{H}-\Delta\right)+\epsilon p_{H}\left(\alpha \Delta_{H}-\mu\right) \\
\frac{d p_{D}}{d \tau}=p y\left(\Delta_{D}-\Delta\right)+\epsilon p_{D}\left(\alpha \Delta_{D}-\mu\right)
\end{array}\right.
$$

When $\varepsilon=0$, system (2.4) gives rise to the fast equation:

$$
\frac{d x}{d \tau}=\frac{x}{2}(1-x)(G-C x) .
$$

The equilibria of equation (2.5) are $x=0, x=1$ and $x=G / C$ whenever $0<G / C<1$.

In order to aggregate, we make the assumption that the fast process is at the fast equilibrium. Thus, we come back to the complete initial system (2.4), substitute the previous fast equilibrium and add the two predator equations. As a consequence, according to the prey density, we obtain two different aggregated models which are valid on two domains of the phase plane:

Model I: $n<\frac{C}{a}, x^{*}=\frac{a n}{C}$ is asymptotically stable in Eq.(2.5),

$$
\left\{\begin{array}{l}
\frac{d n}{d t}=r n\left(1-\frac{n}{K}\right)-a n p, \\
\frac{d p}{d t}=-\mu p+\frac{\alpha a}{2} n p-\frac{\alpha a^{2}}{2 C} n^{2} p .
\end{array}\right.
$$

Model II: $n>\frac{C}{a}, x^{*}=1$ is asymptotically stable in Eq. (2.5),

$$
\left\{\begin{array}{l}
\frac{d n}{d t}=r n\left(1-\frac{n}{K}\right)-a n p, \\
\frac{d p}{d t}=-\mu p+\frac{\alpha a}{2} n p-\frac{\alpha C}{2} p .
\end{array}\right.
$$

These two models connect at the vertical line $n=C / a$ of the phase space $(n, p)$.

The analysis of the "aggregated" models (see [12]) showed that under some conditions, several prey-predator stable equilibria can coexist. The main conclusion of this work was the existence of a relationship between prey density and the strategy adopted by predators: aggressive behaviour is connected to high prey and low predator densities, whereas a polymorphism dove-hawk is found at low prey and high predator densities.

The model was extended to the case of a detailed sequence of individual behaviours such as searching, finding and handling the prey by the predators as well as the intra-specific fighting between the predators, (see Auger et al. [13]). At time $t$, the predator population is divided into six classes: searching and dove predators $\left(p_{S D}\right)$, finding and dove predators $\left(p_{F D}\right)$, defending and dove predators $\left(p_{D D}\right)$, searching and hawk predators $\left(p_{S H}\right)$, finding and hawk predators $\left(p_{F H}\right)$ and defending and hawk predators $\left(p_{D H}\right)$. The individuals can change their tactics only in the defending subpopulation. The fluxes between the 
hawk and dove behaviours are given by the gain matrix (2.1) according to the hawk-dove model. The analysis of the aggregated model by bifurcation methods (see [13]) showed complex global prey-predator dynamics. The main result of this analysis was that in a narrow area of fighting cost values between hawk predators, a stable equilibrium and a stable limit cycle separated by an unstable limit cycle could coexist. Therefore, according to initial conditions, the prey and predator populations can coexist at constant biomasses or cycle.

We also mention a model of predator and prey grouping strategies using game theory, (see [23]). In the predator-prey system they considered, predators are assumed to adopt two strategies, either cooperating with conspecifics to attack preys (strategy $C$ ) or hunting solitary (strategy $S$ ). Preys can also adopt two strategies, either aggregating, i.e., staying with conspecifics to form groups (strategy $A$ ), or remaining lonely (strategy $L$ ). The game model considered the benefits and costs of being in a group for hunting predators and foraging prey. Prey grouped individuals faced less predation but had less access to the resource shared with other individuals of the group. Groups of predators were more efficient to catch prey than solitary ones but shared prey with predators of the group. Under some conditions, it was shown that the proportions of grouped vs. solitary predators and preys could be periodic solutions (see $[23])$.

In Mchich et al. [29], the previous fast theoretical game dynamics was coupled to a slow predator-prey dynamics model using the same method with two time scales as before. The main result of this work was that lonely preys could exclude grouped predators and that predators could coexist against grouped preys, when using aggregative strategy.

It also makes sense to think that individuals of different species competing for the same food source may use aggressive tactics to avoid individuals of another species to get an access to some common food source. In Auger et al. [15], a hawk-dove game model was proposed and analyzed with two populations in which individuals could use hawk and dove tactics. In this model, individuals could fight against individuals of their own population and with individuals of another population. The gains obtained by hawk and dove individuals of population $i$ when interacting with hawk and dove individuals of population $j$ are represented by matrix $\mathbf{A}_{i j}$ which is a classical hawk-dove matrix:

$$
\mathbf{A}_{i j}=\left(\begin{array}{cc}
\frac{G-C_{i j}}{2} & G \\
0 & \frac{G}{2}
\end{array}\right)
$$

where $C_{12}$ (resp. $C_{21}$ ) is the cost when a hawk of population 1 (resp. 2) fights against a hawk of population 2 (resp. 1). If we call $\mathbf{A}$ the following matrix:

$$
\mathbf{A}=\left(\begin{array}{cc}
w_{1} \mathbf{A}_{11} & \left(1-w_{1}\right) \mathbf{A}_{12} \\
\left(1-w_{2}\right) \mathbf{A}_{21} & w_{2} \mathbf{A}_{22}
\end{array}\right)
$$

where $w_{i}, i=1,2$ could be considered as the relative intra-population $i$ encounter rate. Then, the game dynamics is written according to the replicator equation and describes the change of the $H$ and $D$ proportions in each population as follows:

$$
\left\{\begin{array}{l}
\frac{d x_{1}^{H}}{d \tau}=x_{1}^{H}\left((1,0,0,0) \mathbf{A} x^{T}-x_{1} \mathbf{A} x^{T}\right), \\
\frac{d x_{1}^{D}}{d \tau}=x_{1}^{D}\left((0,1,0,0) \mathbf{A} x^{T}-x_{1} \mathbf{A} x^{T}\right), \\
\frac{d x_{2}^{H}}{d \tau_{D}}=x_{2}^{H}\left((0,0,1,0) \mathbf{A} x^{T}-x_{2} \mathbf{A} x^{T}\right), \\
\frac{d x_{2}^{D}}{d \tau}=x_{2}^{D}\left((0,0,0,1) \mathbf{A} x^{T}-x_{2} \mathbf{A} x^{T}\right),
\end{array}\right.
$$

where $x_{1}^{H}$ and $x_{1}^{D}$ (resp. $x_{2}^{H}$ and $x_{2}^{D}$ ) are the proportion of hawks and doves in the total population 1 (resp. $2): x_{i}^{J}=\frac{n_{i}^{J}}{n_{i}}, i \in\{1,2\}, J \in\{H, D\}$ and $x=\left(x_{1}^{H}, x_{1}^{D}, x_{2}^{H}, x_{2}^{D}\right), x_{1}=\left(x_{1}^{H}, x_{1}^{D}, 0,0\right), x_{2}=\left(0,0, x_{2}^{H}, x_{2}^{D}\right)$. 
One must note that $x_{1}^{H}+x_{1}^{D}=1$ and $x_{2}^{H}+x_{2}^{D}=1$. Consequently, system (2.8) reduces to the next two equations:

$$
\left\{\begin{array}{l}
\frac{d x}{d t}=\frac{x}{2}(1-x)\left(G-w_{1} C_{11} x-\left(1-w_{1}\right) C_{12} y\right) \\
\frac{d y}{d t}=\frac{y}{2}(1-y)\left(G-\left(1-w_{2}\right) C_{21} x-w_{2} C_{22} y\right)
\end{array}\right.
$$

in which $x=x_{1}^{H}$ and $y=x_{2}^{H}$. In Auger et al. [15], the analysis of the hawk-dove two populations model is made and shows multiple possibilities of stable equilibria according to parameters values.

In Sanchez et al. [37], the previous fast theoretical game dynamics was coupled to a slow two populations dynamics model. In this work, the fast part of the model describing the game dynamics was given by:

$$
\mathbf{A}=\left(\begin{array}{cccc}
\frac{G-C}{2} & G & \frac{G-C_{12}}{2} & G \\
0 & \frac{G}{2} & 0 & \frac{G}{2} \\
\frac{G-C_{21}}{2} & G & \frac{G-C}{2} & G \\
0 & \frac{G}{2} & 0 & \frac{G}{2}
\end{array}\right)
$$

where the $\operatorname{cost} C$ due to injuries of hawks conflicts are assumed identical within both population. For each sub-population ( $H$ and $D$ ) of any population, the slow part is composed of two terms, linear growth term and negative quadratic terms taking into account long term negative effects of encounters. The analysis has shown multiple equilibria that can be stable or not under conditions on parameters.

We also refer to Moussaoui et al. [31] in which the authors coupled a fast hawk-dove game model similar to Auger et al. [15] but assuming that only inter-population fightings occurred:

$$
\left\{\begin{aligned}
\frac{d n_{1}^{H}}{d \tau}= & n_{1} x_{1}^{H}\left((1,0,0,0) \mathbf{A} x^{T}-x_{1} \mathbf{A} x^{T}\right) \\
& +\varepsilon\left(r_{1} n_{1}^{H}\left(1-\frac{n_{1}}{K_{1}}\right)-n_{1}^{H}\left(k_{12}^{H} n_{2}^{H}+k_{12}^{H D} n_{2}^{D}\right)\right) \\
\frac{d n_{1}^{D}}{d \tau}= & n_{1} x_{1}^{D}\left((0,1,0,0) \mathbf{A} x^{T}-x_{1} \mathbf{A} x^{T}\right) \\
& +\varepsilon\left(r_{1} n_{1}^{D}\left(1-\frac{n_{1}}{K_{1}}\right)-n_{1}^{D}\left(k_{12}^{D H} n_{2}^{H}+k_{12}^{D} n_{2}^{D}\right)\right) \\
\frac{d n_{2}^{H}}{d \tau}= & n_{2} x_{2}^{H}\left((0,0,1,0) \mathbf{A} x^{T}-x_{2} \mathbf{A} x^{T}\right) \\
& +\varepsilon\left(r_{2} n_{2}^{H}\left(1-\frac{n_{2}}{K_{2}}\right)-n_{2}^{H}\left(k_{21}^{H} n_{1}^{H}+k_{21}^{H D} n_{1}^{D}\right)\right) \\
\frac{d n_{2}^{D}}{d \tau}= & n_{2} x_{2}^{D}\left((0,0,0,1) \mathbf{A} x^{T}-x_{2} \mathbf{A} x^{T}\right) \\
& +\varepsilon\left(r_{2} n_{2}^{D}\left(1-\frac{n_{2}}{K_{2}}\right)-n_{2}^{D}\left(k_{21}^{D H} n_{1}^{H}+k_{21}^{D} n_{1}^{D}\right)\right)
\end{aligned}\right.
$$

The first term in right side of system (2.10) represents the game dynamics while the second term corresponds to demography. $r_{i}$ is the growth rate of species $i$ : At each particular encounter, the winner of the game gains an access to a common resource that is quantified by a gain $G_{i}$ that is translated into a greater growth rate $r_{i}$ :

$$
r_{i}=\gamma G_{i}
$$

where $\gamma$ is a positive constant which represents a conversion coefficient of the gain $G_{i}$ of the resource into biomass of the individual. As in the previous model, the negative quadratic terms take into account the long term effects of encounters between individuals playing the same or different tactics on the growth of the population. 


\begin{tabular}{|l|c|}
\hline Fast equilibrium point & Domain of local asymptotic stability \\
\hline \hline$(0,0)$ & $\emptyset$ \\
\hline$(1,0)$ & $G_{2}<C_{21}$ \\
\hline$(0,1)$ & $G_{1}<C_{12}$ \\
\hline$(1,1)$ & $G_{2}>C_{21}$ and $G_{1}>C_{12}$ \\
\hline$\left(\frac{G_{1}}{C_{12}}, \frac{G_{2}}{C_{21}}\right)$ & $\emptyset$ \\
\hline
\end{tabular}

Table 1. Equilibrium points for the fast system and their domains of stability.

According to game theory [38], the payoff matrix A reads:

$$
\mathbf{A}=\left(\begin{array}{cccc}
0 & 0 & \frac{G_{1}-C_{12}}{2} & G_{1} \\
0 & 0 & 0 & \frac{G_{1}}{2} \\
\frac{G_{2}-C_{21}}{2} & G_{2} & 0 & 0 \\
0 & \frac{G_{2}}{2} & 0 & 0
\end{array}\right)
$$

From the complete system (2.10), we applied aggregation methods [14] to obtain an aggregated system, governing the two total species densities. The aggregation of the complete model consists in supposing that the fast dynamics, that is game dynamics, has already attained its stable equilibrium $\left(x^{*}, y^{*}\right)$ (see Table 1), so we can make the following substitutions in system (2.10): $n_{1}^{H}=x^{*} n_{1}, n_{2}^{H}=y^{*} n_{2}$ and then by adding up the two first equations and the two last equations we obtain (after normalization; $\left.u=\frac{n_{1}}{K_{1}}, v=\frac{n_{2}}{K_{2}}\right)$

$$
\left\{\begin{array}{l}
\frac{d u}{d t}=r_{1} u(1-u-a v) \\
\frac{d v}{d t}=r_{2} v(1-v-b u)
\end{array}\right.
$$

The aggregated model $(2.11)$ is a classical interspecific Lotka-Volterra model $[18,33]$ in which parameters read as the following expressions:

$$
a=\alpha_{1} \frac{K_{2}}{r_{1}}, b=\alpha_{2} \frac{K_{1}}{r_{2}}
$$

where

$$
\alpha_{1}=\beta\left(\left(1+y^{*}-x^{*}\right) \frac{G_{1}}{2}+\frac{C_{12}}{2} x^{*} y^{*}\right), \alpha_{2}=\beta\left(\left(1+x^{*}-y^{*}\right) \frac{G_{2}}{2}+\frac{C_{21}}{2} x^{*} y^{*}\right)
$$

and $\beta$ is a positive parameter which represents an encounter rate. An interesting result of this paper

\begin{tabular}{|c|c|c|c|c|}
\hline & $\begin{array}{l}\text { Fast } \\
\text { equilibrium } \\
\text { point }\end{array}$ & $\begin{array}{l}\text { Competive } \\
\text { coefficient }\end{array}$ & $\begin{array}{l}\text { Equilibrium } \\
\text { point }\end{array}$ & Local stability \\
\hline Model 1 & $(1,0)$ & $\begin{array}{l}a=0 \\
b=\frac{\beta}{\gamma} K_{1}\end{array}$ & $\begin{array}{l}(0,0) \\
(1,0) \\
(0,1) \\
(1,1-b)\end{array}$ & $\begin{array}{l}\text { unstable point } \\
\text { stable exclusion if } b>1 \\
\text { saddle point } \\
\text { stable coexistence }\end{array}$ \\
\hline Model 2 & $(0,1)$ & $\begin{array}{l}a=\frac{\beta}{\gamma} K_{2} \\
b=0\end{array}$ & $\begin{array}{l}(0,0) \\
(1,0) \\
(0,1) \\
(1-a, 1)\end{array}$ & $\begin{array}{l}\text { unstable point } \\
\text { saddle point } \\
\text { stable exclusion if } a>1 \\
\text { stable coexistence }\end{array}$ \\
\hline Model 3 & $(1,1)$ & $\begin{array}{l}a=\frac{\beta}{\gamma} K_{2}\left(1+\frac{C_{12}}{G_{1}}\right) \\
b=\frac{\beta}{\gamma} K_{1}\left(1+\frac{C_{21}}{G_{2}}\right)\end{array}$ & $\begin{array}{l}(0,0) \\
(1,0) \\
(0,1) \\
\left(\frac{1-a}{1-a b}, \frac{1-b}{1-a b}\right)\end{array}$ & $\begin{array}{l}\text { unstable point } \\
\text { stable exclusion if } b>1 \\
\text { stable exclusion if } a>1 \\
\text { stable coexistence } \\
\text { if } a<1 \text { and } b<1\end{array}$ \\
\hline
\end{tabular}
was that two competing species using pure hawk strategies at the same time can coexist which is in a certain sense counter intuitive as two much aggressiveness in populations can lead to important injuries and exceeding extra-mortality (see Table 2).

Table 2. Conditions for existence and local stability of equilibria of system (2.11) under different situations. 


\section{Model of a stage-structured population with mature individuals using hawk, dove and bully tactics}

In this section, we shall present an original model describing a stage-structured population with individuals using three tactics. To start, let us recall that Auger and Pontier [11] presented an approach of a population modeling that can be used to investigate the influence of intra-individual variability in the behaviour on population dynamics. This approach is based on coupling of a game model describing encounters to a model describing the population dynamics: The same individual can adopt two different tactics: hawk and dove in his life and it is assumed that the game is played at a fast time scale in comparison to population dynamics. This fast part of the model is coupled to a slow part, which describes the growth of each sub-population. This model was applied to the case of cat populations showing that the total population follows a logistic growth in the long term with a carrying capacity decreasing with the proportion of hawks as expected. The model was extended to a hawk-Dove-Bully game [36]. The model showed a similar result with slightly small differences, again in agreement with observations of cat populations. Marvá et al. [25] studied a nonlinear two time scale discrete population model that couples age-structured demography with individual competition for resources. Individuals are divided into juvenile and adult classes, and demography is described by means of a density-dependent Leslie matrix (see [17] for linear case). Adults compete to access resources; every time two adults meet, they choose either being aggressive (hawk) or non aggressive (dove) to get the best payoff. Individual encounters occur much more frequently than demographic events, what yields that the model takes the form of a two time scale system. Approximate aggregation methods allowed to reduce the system while preserving at the same time crucial asymptotic information for the whole population. In this way, they were able to describe the total population size as function of individual aggressiveness level and environmental richness. The authors analyzed the effects of conflicting tactics of resource acquisition on population dynamics. A continuous version of these model was studied in [32], where the authors considered a population subdivided into two distinct stages (immature and mature) and they assumed that mature individuals acquire resources required to survive and reproduce by using two contrasted behavioural tactics (hawk versus dove). The analysis of the reduced model showed that proportion of hawks in less aggressive populations, i.e. when individuals fight at small cost with few injuries, is expected to be larger than in aggressive populations fighting at large cost. The maximum of the total density at equilibrium in a rich environment was smaller for larger costs.

In this section, we consider a population subdivided into two distinct stages: immature and mature. The immature sub-population do not participate to reproduction. The mature individuals are assumed to compete to access resource required for survive and reproduce. Mature reproductive individuals adults choose between hawk $(H)$, dove $(D)$ and bully $(B)$ tactics in order to get access to a resource. Hawk individuals are always aggressive. Dove individuals are not aggressive and they never escalate. Bully individuals start by escalating but retreat if opponent escalates. These three behavioural phenotypes are assumed to be achievable to all individuals, whatever their genotypes. Individuals may choose these alternative tactics according to the conspecific encountered (physical strength, dominance rank of the opponent). Let $n_{1}(t)$ be the size of the immature individuals and $n_{2}(t)$ the total size of the mature individuals population at time $t$. The model is composed of two parts, a fast part that describes the change of tactics of mature and a slow part that describes the demographic process. We are going to present these two parts by using two sub-models as in following:

\subsection{Fast part: hawk-dove-bully game}

We assume that individuals frequently encounter each other and compete for resources (food or mates). This process is going on at a fast time scale compared to the long term evolution of the population. We assume that three behavioural phenotypes, hawk $(H)$, dove $(D)$ and bully $(B)$ are present within the population. The state of a population concerning this conflict is fully described by a vector $x=$ $\left[x_{H}, x_{D}, x_{B}\right]^{T} \in \mathbb{R}^{3}$ where $u^{T}$ means the transpose of vector $u$ and $x_{H}=n_{2}^{H} / n_{2}, x_{D}=n_{2}^{D} / n_{2}, x_{B}=$ 
$n_{2}^{B} / n_{2}$ where $n_{2}^{H}, n_{2}^{D}, n_{2}^{B}$ are, respectively, the number of hawk, dove and bully and $n_{2}=n_{2}^{H}+n_{2}^{D}+n_{2}^{B}$. Consequently, $x_{i} \geq 0$ for all $i$ and $\sum_{i \in \mathcal{A}} x_{i}=1$, where $\mathcal{A}=\{H, D, B\}$.

Let $\mathbf{A}$ be the $3 \times 3$ matrix representing the payoff. Then the payoff of tactic $I$ against tactic $J$ is $a_{I J}$ for $I, J \in \mathcal{A}$, the payoff to tactic $I$ against population $x$ is $(\mathbf{A} x)_{I}=\sum_{J \in \mathcal{A}} a_{I J} x_{J}$ and the payoff to population $x$ against itself is $x \mathbf{A} x=\sum_{I \in \mathcal{A}} x_{I}(\mathbf{A} x)_{I}$. We are using as $\mathbf{A}$, the following classical $H-D-B$ payoff matrix $[22,28,38,41]$

$$
\mathbf{A}=\left(\begin{array}{ccc}
\frac{G-C}{2} & G & G \\
0 & \frac{G}{2} & 0 \\
0 & G & \frac{G}{2}
\end{array}\right),
$$

where $G$ is the gain, $C$ is the cost of injuries resulting of fighting between aggressive individuals. The game dynamics, played at the fast time scale, is written according to the replicator equations $[22,26,27]$, based upon the main hypothesis that the rate of growth of those playing tactic $I$ is proportional to the advantage of $I$. By suitable choice of time scale we can write:

$$
\left\{\begin{array}{l}
\frac{d x_{H}}{d t}=x_{H}\left((\mathbf{A} x)_{H}-x \mathbf{A} x\right), \\
\frac{d x_{D}}{d t}=x_{D}\left((\mathbf{A} x)_{D}-x \mathbf{A} x\right), \\
\frac{d x_{B}}{d t}=x_{B}\left((\mathbf{A} x)_{B}-x \mathbf{A} x\right) .
\end{array}\right.
$$

These equations assume that an individual compares the gain of each pure tactic to the average gain. If a tactic leads to a larger payoff than the average one, the proportion of individuals of the population using this tactic is increasing.

The analysis of system (3.1) (see [41]) in $\Omega=\left\{x \in \mathbb{R}^{3}: x_{I} \geq 0, \sum_{I} x_{I}=1\right\}$, when $G<C$ gives us the existence of single point attractor, $x^{*}=(G / C, 0,1-G / C)^{T}$, which essentially determine the asymptotic behaviour of solutions. Thus, we have a globally stable equilibrium, the polymorphism of $G / C$ hawk and $1-G / C$ bully tactic. When $G>C$, the stationary point $x^{*}=(1,0,0)$ is asymptotically stable, the population is monomorphic (there are only Hawks).

\subsection{Slow part: demography}

The assumptions of this model are as follows:

1. The birth rate into the immature population is proportional to the existing mature population with a fecundity coefficient that depends on average gains $\bar{G}_{i}, i \in \mathcal{A} ; \mu_{1}$ is the death rate of the immature population. We also assume that the immature individual survival is density-dependent with the effect coefficient $-\eta$.

2. The transformation rate from the immature individuals to mature individuals is proportional to the existing immature population with proportionality $\beta$. For the mature equations, we assume that escalated contests cause injuries and therefore provoke an increase of the mortality of mature individuals according to the following relationship: $\mu_{2}\left(\bar{C}_{i}\right)=\mu+\alpha \bar{C}_{i}$, where $\mu$ is the constant natural mortality rate supposed identical for hawks, doves and bully, $\bar{C}_{i}$ is the average cost, $i \in \mathcal{A}$ and $\alpha$ a positive coefficient which permits to regulate the effect of the average cost on the survival.

$$
\left\{\begin{array}{l}
\frac{d n_{1}}{d t_{H}}=F_{H}\left(\bar{G}_{H}\right) n_{2}^{H}+F_{D}\left(\bar{G}_{D}\right) n_{2}^{D}+F_{B}\left(\bar{G}_{B}\right) n_{2}^{B}-\mu_{1} n_{1}-\beta n_{1}-\eta n_{1}^{2}, \\
\frac{d n_{2}^{H}}{d t_{D}}=q_{H} \beta n_{1}-\mu_{2}\left(\bar{C}_{H}\right) n_{2}^{H}, \\
\frac{d n_{2}}{d t_{B}}=q_{D} \beta n_{1}-\mu_{2}\left(\bar{C}_{D}\right) n_{2}^{D}, \\
\frac{d t}{d t}=q_{B} \beta n_{1}-\mu_{3}\left(\bar{C}_{B}\right) n_{2}^{B} .
\end{array}\right.
$$

Parameters $q_{i}$ represent the proportion of immature individuals becoming hawks, doves and bully when surviving to mature state (with $\sum_{i \in \mathcal{A}} q_{i}=1$ ).

The average cost is calculated by adding the cost of each type of encounter weighted by the proportion of 
this type of encounter. Therefore, the average cost received by a dove, that we note $\bar{C}_{D}$ is null, because doves do not fight and do not get injured:

$$
\bar{C}_{D}=0 \frac{n_{2}^{H}}{n_{2}}+0 \frac{n_{2}^{D}}{n_{2}}+0 \frac{n_{2}^{B}}{n_{2}}=0,
$$

it is the same for the average cost received by a bully, that we note $\bar{C}_{B}$, indeed;

$$
\bar{C}_{B}=0 \frac{n_{2}^{H}}{n_{2}}+0 \frac{n_{2}^{D}}{n_{2}}+0 \frac{n_{2}^{B}}{n_{2}}=0,
$$

as a consequence, dove mortality and bully mortality are simply $\mu$.

$$
\mu_{2}\left(\bar{C}_{D}\right)=\mu_{2}\left(\bar{C}_{B}\right)=\mu .
$$

On the contrary, hawks fight and get injured which causes them higher mortality risks than doves. Hawk mortality is a function of the average cost received by a hawk, that we note $\bar{C}_{H}$ :

$$
\bar{C}_{H}=\left(\frac{C}{2}\right) \frac{n_{2}^{H}}{n_{2}}+0 \frac{n_{2}^{D}}{n_{2}}+0 \frac{n_{2}^{B}}{n_{2}}=\left(\frac{C}{2}\right) \frac{n_{2}^{H}}{n_{2}} .
$$

The hawk mortality $\mu_{2}\left(\overline{C_{H}}\right)$ is thus given by the following expression:

$$
\mu_{2}\left(\bar{C}_{H}\right)=\mu+\alpha\left(\frac{C}{2}\right) \frac{n_{2}^{H}}{n_{2}} .
$$

\subsubsection{Fecundity}

For some species, the amount of resource has a continuous effect on fecundity, while for others, individuals must accumulate a given amount of resource before reproduction. Average gains are calculated by adding the gain of each type of encounter weighted by the proportion of this type of encounter. Thus, we obtain the next expressions for hawk $(H)$, dove $(D)$ and bully $(B)$ average gains:

$$
\begin{aligned}
\bar{G}_{H} & =\left(\frac{G}{2}\right) \frac{n_{2}^{H}}{n_{2}}+G \frac{n_{2}^{D}}{n_{2}}+G \frac{n_{2}^{B}}{n_{2}}, \\
\bar{G}_{D} & =0 \frac{n_{2}^{H}}{n_{2}}+\frac{G}{2} \frac{n_{2}^{D}}{n_{2}}+0 \frac{n_{2}^{B}}{n_{2}}=\left(\frac{G}{2}\right) \frac{n_{2}^{D}}{n_{2}}, \\
\bar{G}_{B} & =0 \frac{n_{2}^{H}}{n_{2}}+G \frac{n_{2}^{D}}{n_{2}}+\frac{G}{2} \frac{n_{2}^{B}}{n_{2}}=G \frac{n_{2}^{D}}{n_{2}}+\frac{G}{2} \frac{n_{2}^{B}}{n_{2}} .
\end{aligned}
$$

In order to study the effect of the resource on fecundity, we use a Holling type function. According to this function, fecundity increases with the average gain and then reaches a plateau. The average gain represents an average energy that can be used by the individual. This fecundity function assumes that when the average gain is small, it is mainly used for maintenance. When it becomes larger, it can also be used for fecundity to produce offsprings with a maximum corresponding to the plateau. Thus, we choose the following general relationship between fecundity and average gain:

$$
H\left(\bar{G}_{i}\right)=F \frac{\bar{G}_{i}}{\gamma+\bar{G}_{i}} \text { where } i \in \mathcal{A} .
$$

We use the average gains of Hawk, Dove and Bully of Eqs. (3.3), (3.4) and (3.5) to calculate their respective fecundities. Fecundity is an increasing function of the average gain. Parameter $\gamma$ permits to influence the "speed" to reach the plateau of the fecundity. When $\gamma$ decreases the maximum of the fecundity is reached more quickly. 


\subsection{The complete slow-fast model}

The complete model is obtained by coupling the previous hawk-dove-bully behavioural model and the populations model as follows:

$$
\left\{\begin{array}{l}
\varepsilon \frac{d n_{1}}{d t}=\varepsilon\left(F\left(\frac{\bar{G}_{H}}{\gamma+\bar{G}_{H}} n_{2}^{H}+\frac{\bar{G}_{D}}{\gamma+\bar{G}_{D}} n_{2}^{D}+\frac{\bar{G}_{B}}{\gamma+\bar{G}_{B}} n_{2}^{B}\right)-\left(\mu_{1}+\beta\right) n_{1}-\eta n_{1}^{2}\right), \\
\varepsilon \frac{d n_{2}^{H}}{d t}=n_{2} x_{H}\left(\Delta_{H}-\Delta\right)+\varepsilon\left(q_{H} \beta n_{1}-\left(\mu+\alpha \frac{C}{2} \frac{n_{2}^{H}}{n_{2}}\right) n_{2}^{H}\right), \\
\varepsilon \frac{d n_{2}^{D}}{d t_{B}}=n_{2} x_{D}\left(\Delta_{D}-\Delta\right)+\varepsilon\left(q_{D} \beta n_{1}-\mu n_{2}^{D}\right), \\
\varepsilon \frac{d n_{2}^{B}}{d t}=n_{2} x_{B}\left(\Delta_{B}-\Delta\right)+\varepsilon\left(q_{B} \beta n_{1}-\mu n_{2}^{B}\right),
\end{array}\right.
$$

where $\varepsilon$ is a small parameter. It is also usual to use the fast time scale $\tau$

$$
\left\{\begin{array}{l}
\frac{d n_{1}}{d \tau}=\varepsilon\left(F\left(\frac{\bar{G}_{H}}{\gamma+\bar{G}_{H}} n_{2}^{H}+\frac{\bar{G}_{D}}{\gamma+\bar{G}_{D}} n_{2}^{D}+\frac{\bar{G}_{B}}{\gamma+\bar{G}_{B}} n_{2}^{B}\right)-\left(\mu_{1}+\beta\right) n_{1}-\eta n_{1}^{2}\right), \\
\frac{d n_{2}^{H}}{d \tau}=n_{2} x_{H}\left(\Delta_{H}-\Delta\right)+\varepsilon\left(q_{H} \beta n_{1}-\left(\mu+\alpha \frac{C}{2} \frac{n_{2}^{H}}{n_{2}}\right) n_{2}^{H}\right), \\
\frac{d n_{2}^{D}}{d \tau_{B}}=n_{2} x_{D}\left(\Delta_{D}-\Delta\right)+\varepsilon\left(q_{D} \beta n_{1}-\mu n_{2}^{D}\right), \\
\frac{d n_{2}^{B}}{d \tau}=n_{2} x_{B}\left(\Delta_{B}-\Delta\right)+\varepsilon\left(q_{B} \beta n_{1}-\mu n_{2}^{B}\right) .
\end{array}\right.
$$

In this last form, it is obvious that the game dynamics correspond to the fast time scale while the small terms of the order of $\varepsilon$ correspond to the slow time scale. This model is a four-dimensional system of ordinary differential equations.

\section{The aggregated model}

From the complete system (3.7), we apply aggregation methods to obtain an aggregated model, i.e. a system of differential equation governing the total immature and mature populations. Aggregation methods permit to reduce a large system of equations involving different time scales into a smaller system with a few global variables. The method is based on perturbation technics [8-10].

According to parameters values, two cases can occur:

- $G<C, x^{*}=(G / C, 0,1-G / C)$ is asymptotically stable for system (3.1) and this for any initial condition $x(0)$ such that $0<x_{H}(0)+x_{D}(0)+x_{B}(0) \leq 1$. In this case, at equilibrium, the population is polymorphic with a proportion $\frac{G}{C}$ of hawks and $1-\frac{G}{C}$ of bully and without doves. When replacing the variables $n_{2}^{H}, n_{2}^{D}$ and $n_{2}^{B}$ by those of the fast equilibrium, the average costs and gains become:

$$
\begin{aligned}
& \bar{C}_{H}=\frac{G}{2}, \quad \bar{C}_{D}=0, \quad \bar{C}_{B}=0, \\
& \bar{G}_{H}=G\left(1-\frac{G}{2 C}\right), \quad \bar{G}_{D}=0, \quad \bar{G}_{B}=\frac{G}{2}\left(1-\frac{G}{C}\right) .
\end{aligned}
$$

- $G>C, x^{*}=\left(\frac{G}{C}, 0,1-\frac{G}{C}\right)$ does not belong to $\Omega$. The equilibrium $x^{*}=(1,0,0)$ is asymptotically stable for system (3.1). The population is monomorphic and totally hawk at equilibrium. When replacing the variables $n_{2}^{H}, n_{2}^{D}$ and $n_{2}^{B}$ of the complete model by those of the fast equilibrium, the average costs and gains become:

$$
\begin{array}{lll}
\bar{C}_{H}=\frac{C}{2}, & \bar{C}_{D}=0, & \bar{C}_{B}=0, \\
\bar{G}_{H}=\frac{G}{2}, & \bar{G}_{D}=0, & \bar{G}_{B}=0 .
\end{array}
$$

In order to aggregate, we make the assumption that the fast process is at the fast equilibrium. Thus, we come back to the complete initial system (3.7), substitute the previous fast equilibrium and add the three 
last equations. It is necessary to replace the fast variables in terms of the fast equilibrium as follows:

$$
n_{2}^{H}=x_{H}^{*} n_{2}, n_{2}^{D}=x_{D}^{*} n_{2} \text { and } n_{2}^{B}=x_{B}^{*} n_{2} .
$$

After a straightforward calculation, one obtains the following system of two equations governing the immature and mature densities at the slow time scale, that we call the aggregated model

$$
\left\{\begin{array}{l}
\frac{d n_{1}}{d t}=F\left(\frac{\bar{G}_{H}}{\gamma+\bar{G}_{H}} x_{H}^{*} n_{2}+\frac{\bar{G}_{D}}{\gamma+\bar{G}_{D}} x_{D}^{*} n_{2}+\frac{\bar{G}_{B}}{\gamma+\bar{G}_{B}} x_{B}^{*} n_{2}\right)-\left(\mu_{1}+\beta\right) n_{1}-\eta n_{1}^{2}, \\
\frac{d n_{2}}{d t}=\beta n_{1}-\mu n_{2}-\alpha \frac{C}{2}\left(x_{H}^{*}\right)^{2} n_{2} .
\end{array}\right.
$$

The dynamics of the aggregated system (4.1) is a good approximation of the dynamics of the complete system (3.7) provided the aggregated system is structurally stable, which is the case (see section 4.1 for the analysis); and $\varepsilon$ is small enough, which is assumed. In our case, we remember that we can have two possibilities for the fast equilibrium, then we obtain two different aggregated models which are valid on two domains of the phase plane:

- Model I: $G<C, x^{*}=\left(\frac{G}{C}, 0,1-\frac{G}{C}\right)$ is asymptotically stable for system (3.1),

$$
\left\{\begin{array}{l}
\frac{d n_{1}}{d t}=F\left(\frac{\bar{G}_{H}}{\gamma+\bar{G}_{H}} \frac{G}{C}+\frac{\bar{G}_{B}}{\gamma+\bar{G}_{B}}\left(1-\frac{G}{C}\right)\right) n_{2}-\left(\mu_{1}+\beta\right) n_{1}-\eta n_{1}^{2} \\
\frac{d n_{2}}{d t}=\beta n_{1}-\mu n_{2}-\alpha \frac{G^{2}}{2 C} n_{2}
\end{array}\right.
$$

where

$$
\bar{G}_{H}=G\left(1-\frac{G}{2 C}\right), \bar{G}_{B}=\frac{G}{2}\left(1-\frac{G}{C}\right) .
$$

- Model II: $G>C, x^{*}=(1,0,0)$ is asymptotically stable for system (3.1),

$$
\left\{\begin{array}{l}
\frac{d n_{1}}{d t}=F \frac{\bar{G}_{H}}{\gamma+\bar{G}_{H}} n_{2}-\left(\mu_{1}+\beta\right) n_{1}-\eta n_{1}^{2} \\
\frac{d n_{2}}{d t}=\beta n_{1}-\mu n_{2}-\alpha \frac{C}{2} n_{2}
\end{array}\right.
$$

where $\bar{G}_{H}=\frac{G}{2}$.

It must be noted that there is continuity, i.e. average gains and costs in mixed and pure hawk cases become equal at the separation line between the two models, when $G=C$, in this case, these two models connect.

\subsection{Analysis of the aggregated model}

We set up the stage structured model on the general form

$$
\left\{\begin{array}{l}
\frac{d n_{1}}{d t}=F_{1} n_{2}-\left(\mu_{1}+\beta\right) n_{1}-\eta n_{1}^{2} \\
\frac{d n_{2}}{d t}=\beta n_{1}-d n_{2}
\end{array}\right.
$$

where $F_{1}=F\left(\frac{\bar{G}_{H}}{\gamma+\bar{G}_{H}} \frac{G}{C}+\frac{\bar{G}_{B}}{\gamma+\bar{G}_{B}}\left(1-\frac{G}{C}\right)\right), d=\mu+\alpha \frac{G^{2}}{2 C}$ in the case of mixed strategy and $F_{1}=F \frac{\bar{G}_{H}}{\gamma+\bar{G}_{H}}, d=\mu+\alpha \frac{C}{2}$ in the case of pure hawk population. 


\subsubsection{Equilibrium and dissipativness of system (4.4)}

System (4.4) has to be analyzed with the initial conditions $n_{1}(0)>0, n_{2}(0)>0$. We observe that the right-hand side of (4.4) is a smooth function of the variables $\left(n_{1}, n_{2}\right)$; hence, local existence and uniqueness properties are obtained for the corresponding Cauchy problem. The state space of (4.4) remains in the positive octant: $\mathbb{R}_{+}^{2}:=\left\{\left(n_{1}, n_{2}\right): n_{1}>0, n_{2}>0\right\}$.

Indeed, set $\mathbb{R}_{+}^{2}$ is positively invariant since the vector field of (4.4) is inward on the boundary $\partial \mathbb{R}_{+}^{2}$. Now, we shall prove the dissipativness of system (4.4).

Proposition 4.1. All solutions of system (4.4) that start in $\mathbb{R}_{+}^{2}$ are in the compact

$$
\Lambda=\left\{\left(n_{1}, n_{2}\right) \in \mathbb{R}_{+}^{2}: n_{1}+n_{2} \leq \frac{\beta F_{1}}{d \eta}\right\}
$$

Proof. We define the function $w(t)=\frac{n_{1}}{F_{1}}+\frac{n_{2}}{d}$. Therefore,

$$
\frac{d w}{d t} \leq \frac{\beta}{d} n_{1}-\frac{\eta}{F_{1}} n_{1}^{2}
$$

From the standard comparison principle, we get $w \leq z$, where $z$ is the solution of the logistic equation

$$
z^{\prime}=\frac{\beta}{d} z-\frac{\eta}{F_{1}} z^{2}
$$

Hence $w \leq z \leq \limsup _{t \longrightarrow+\infty} z \leq \frac{\beta F_{1}}{d \eta}$. So, we obtain the dissipativness properties of system (4.4).

Now, we investigate non-negative equilibria for system (4.4). The following proposition, gives the existence of equilibria.

Proposition 4.2. System (4.4) has two equilibria:

(i) The trivial equilibrium point $E_{0}(0,0)$.

(ii) The positive equilibrium point $E_{*}\left(n_{1}^{*}, n_{2}^{*}\right)$ exist if and only if $F_{1} \beta>d\left(\mu_{1}+\beta\right)$. Under this condition, $n_{1}^{*}$ and $n_{2}^{*}$ are given by

$$
\begin{aligned}
& n_{1}^{*}=\frac{F_{1} \beta-d\left(\mu_{1}+\beta\right)}{d \eta}, \\
& n_{2}^{*}=\frac{\beta}{d} n_{1}^{*} .
\end{aligned}
$$

Proof. A direct way of finding equilibria is to consider the intersection of the nullclines. We should solve the following algebraic equations:

$$
\begin{aligned}
& F_{1} n_{2}-\left(\mu_{1}+\beta\right) n_{1}-\eta n_{1}^{2}=0, \\
& \beta n_{1}-d n_{2}=0 .
\end{aligned}
$$

The shape of the curves suggests that there is only the trivial point and an interior equilibrium provided that $F_{1} \beta>d\left(\mu_{1}+\beta\right)$. This equilibrium corresponds to the values

$$
\begin{aligned}
n_{1}^{*} & =\frac{F_{1} \beta-d\left(\mu_{1}+\beta\right)}{d \eta}, \\
n_{2}^{*} & =\frac{\beta}{d} n_{1}^{*} .
\end{aligned}
$$




\subsubsection{Dynamic behaviour}

To obtain the local stability results, we use the Jacobian matrix associated with system (4.4)

$$
J\left(n_{1}, n_{2}\right)=\left[\begin{array}{cc}
-\left(\mu_{1}+\beta+2 \eta n_{1}\right) & F_{1} \\
\beta & -d
\end{array}\right] .
$$

Proposition 4.3. (i) If $F_{1} \beta<d\left(\mu_{1}+\beta\right)$, then the origin $E_{0}$ is a stable node.

(ii) If $F_{1} \beta>d\left(\mu_{1}+\beta\right)$, then the origin $E_{0}$ is a saddle point.

Proof. The Jacobian matrix calculated at the origin $E_{0}$ is

$$
J_{0}=\left[\begin{array}{cc}
-\left(\mu_{1}+\beta\right) & F_{1} \\
\beta & -d
\end{array}\right]
$$

It is clear that the trace satisfies

$$
\operatorname{Tr} J_{0}=\lambda_{1}+\lambda_{2}<0
$$

and the determinant

$$
\operatorname{det} J_{0}=\lambda_{1} \lambda_{2}=d\left(\mu_{1}+\beta\right)-F_{1} \beta .
$$

Since a necessary and sufficient condition for local stability in two dimensions is

$$
\operatorname{Tr} J_{0}<0 \text { and } \operatorname{det} J_{0}>0 .
$$

Assertion $(i)$ and $(i i)$ are immediate.

Proposition 4.4. If $F_{1} \beta>d\left(\mu_{1}+\beta\right)$ is verified, then the equilibrium $E_{*}$ given by (4.5) is locally stable.

Proof. The Jacobian matrix calculated at the point $E_{*}$ is

$$
J_{*}=\left[\begin{array}{cc}
-\left(2 \frac{F_{1} \beta}{d}-\left(\mu_{1}+\beta\right)\right) & F_{1} \\
\beta & -d
\end{array}\right] .
$$

It is clear that

$$
\operatorname{Tr} J_{*}=-2 \frac{F_{1} \beta}{d}+\left(\mu_{1}+\beta\right)-d
$$

and

$$
\operatorname{det} J_{*}=F_{1} \beta-d\left(\mu_{1}+\beta\right) .
$$

Hence, if $F_{1} \beta>d\left(\mu_{1}+\beta\right)$, then $\operatorname{Tr} J_{*}<0$ and $\operatorname{det} J_{*}>0$. Therefore, $E_{*}$ is locally stable positive equilibrium.

In the next proposition, we shall show that system (4.1) has no periodic solutions. our method involves application of the Bendixson criterion [2]. The dissipativity of the system and the Poincare- Bendixson theorem [2] imply that the positive solution of system (4.4) tends either to the origin or to $E_{*}\left(n_{1}^{*}, n_{2}^{*}\right)$.

Proposition 4.5. system (4.4) admits no periodic solution. In addition, if $F_{1} \beta<d\left(\mu_{1}+\beta\right)$, then $\left(n_{1}(t), n_{2}(t)\right)$ tends to $(0,0)$. If $F_{1} \beta>d\left(\mu_{1}+\beta\right)$, then $\left(n_{1}(t), n_{2}(t)\right)$ tends to $\left(n_{1}^{*}, n_{2}^{*}\right)$.

Proof. Let

$$
F_{1} n_{2}-\left(\mu_{1}+\beta\right) n_{1}-\eta n_{1}^{2}=P\left(n_{1}, n_{2}\right)
$$

and

$$
\beta n_{1}-d n_{2}=Q\left(n_{1}, n_{2}\right)
$$


Then

$$
\operatorname{div}(P, Q)=\frac{\partial F}{\partial n_{1}}+\frac{\partial Q}{\partial n_{2}}=-\mu_{1}-\beta-2 \eta n_{1}-d,
$$

for all $n_{1} \geq 0$ and $n_{2} \geq 0$, since all other parameters are strictly positive, the sign of $\operatorname{div}(P, Q)$ in (4.7) is strictly negative. Therefore, if we apply the Bendixson criterion [2], there are no limit cycles withing the interior of the positive octant in the state space $\left(n_{1}, n_{2}\right)$. From the Dulac principe [2] and the dissipativness of the system, it follows that $\left(n_{1}(t), n_{2}(t)\right)$ tends either to $(0,0)$ or to $\left(n_{1}^{*}, n_{2}^{*}\right)$.

Proposition 4.6. (i) If $F_{1} \beta<d\left(\mu_{1}+\beta\right)$, then the origin $E_{0}$ is globally asymptotically stable.

(ii) If $F_{1} \beta>d\left(\mu_{1}+\beta\right)$, then the equilibrium $E_{*}$ is globally asymptotically stable.

Proof. It is a direct consequence of Propositions 4.3, 4.4 and 4.5.

It is mathematically interesting to see what happens if $F_{1} \beta=d\left(\mu_{1}+\beta\right)$, since in this case the equilibrium is not hyperbolic and the method of linearization is not conclusive.

Proposition 4.7. If $F_{1} \beta=d\left(\mu_{1}+\beta\right)$, then the unique non negative equilibrium $(0,0)$ is globally asymptotically stable in $\mathbb{R}_{+}^{2}$.

Proof. We construct the following Lyapunov function:

$$
V\left(n_{1}, n_{2}\right)=\beta n_{1}+\left(\mu_{1}+\beta\right) n_{2} .
$$

Calculating the derivative of $V\left(n_{1}, n_{2}\right)$ along (4.4), we obtain

$\frac{d V}{d t}\left(n_{1}, n_{2}\right)=-\beta \eta n_{1}^{2} \leq 0$ in Int $\mathbb{R}_{+}^{2}$.

In addition, $\lim _{n_{1}, n_{2} \rightarrow+\infty} V=+\infty$. This implies that $(0,0)$ is globally asymptotically stable in $\mathbb{R}_{+}^{2}$.

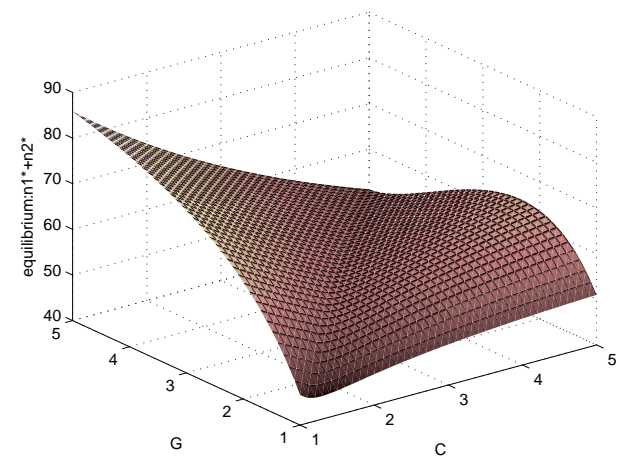

Figure 1. Total density of the population as a function of gain $(G)$ and cost $(C)$ with coefficients: $F=1, \beta=0.6, \mu=0.2, \mu_{1}=0.05, \eta=0.07, \gamma=0.2, \alpha=0.03$. 


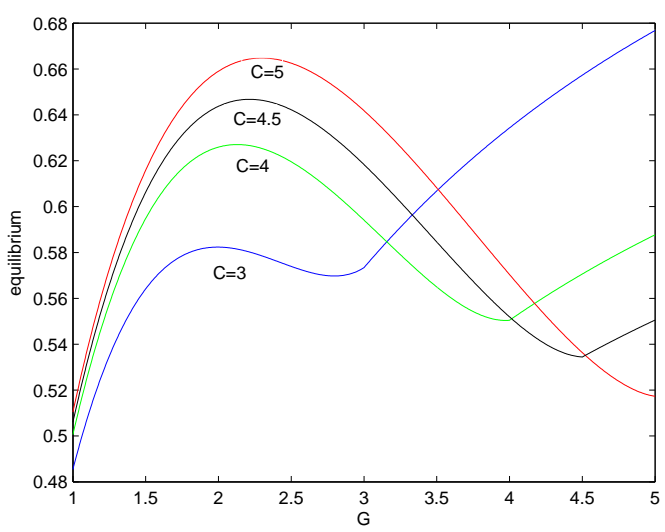

FiguRE 2. Curved line corresponding to maximum population size at equilibirum, the parameters are same as those in Figure 1.

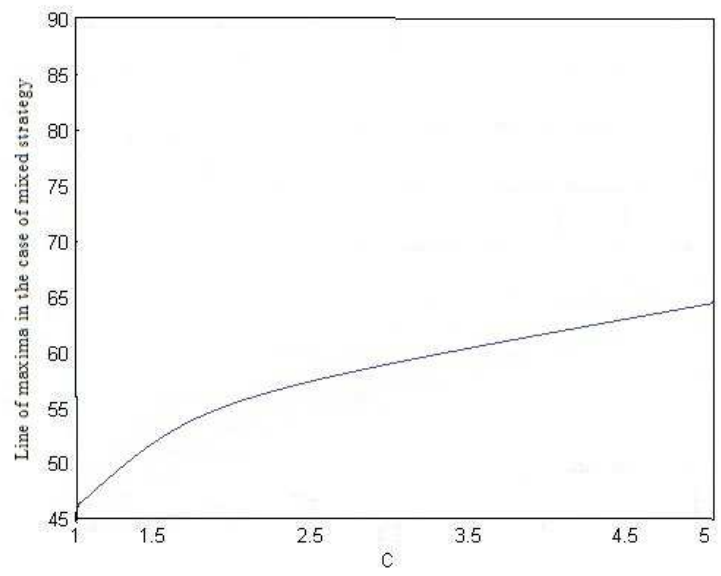

Figure 3. Line of local maxima of the equilibrium population size in the $(G, C)$ plane, the parameters are same as those in Figure 1.

\section{Discussion and Conclusion}

Our aim was to draw conclusions about the effect of some kind of behavioural interactions on population dynamics. The relationship between the equilibrium density of a population and its degree of aggressiveness which was established for the model taking into account two tactics, hawk and dove [32], has been generalized to the hawk-dove-bully game. We obtained the same relationship between the population density at equilibrium and the hawk proportion, as in the classical hawk-dove game [32], but the relationship occurred with a hawk-bully polymorphism instead of a hawk-dove polymorphism. Dove individuals disappear from the population.

We have presented a stage structured population system with mature individuals using hawk, dove and bully tactics. Our model is based on the coupling between population dynamics and game dynamics. This coupling allowed us to describe the overall population dynamics, with individuals using different 


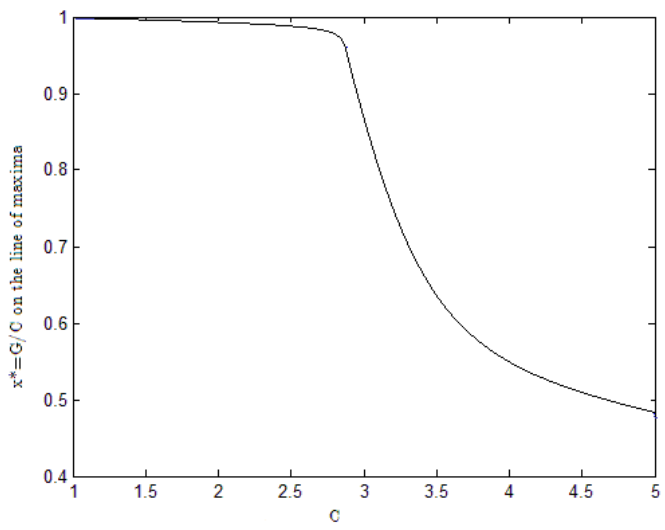

Figure 4. Proportion of hawks along the curved line of maximum population size at equilibrium shown on Figure 1.

tactics during their life and to analyze the effects of different behavioural changes on population growth according to biological processes going on at different time scales (day for contests, year for the demography). Aggregation methods have been applied to the study of dynamical systems with two time scales. In our model, the game operates on a fast-time scale and the demography on a slow-time scale. Assuming that the fast process goes to a stable equilibrium, the aggregated model can be used to evaluate the consequences of changing the proportion of hawks and to study the consequences of changing in individual behavior on population equilibrium.

As a first result, Figure 1 shows the total density of the population, immature and mature, with respect to gain and cost. We can consider that populations having the larger population densities have a better chance to survive in the long term. Regarding game parameters, $C$-values can be considered as a gradient of aggressiveness, from non aggressive species (small $C$ ) to very aggressive, (very large $C)$. $G$-values can represent a gradient of environment, from poor with few resource (small $G$ ) to rich environment (large $G$ ). Figure 1 shows the graph of the total equilibrium population with respect to the gain and the cost with two different domains : " A domain $G<C$ in which the population is mixed with a proportion of hawks $x_{H}^{*}=\frac{G}{C}$ at the fast equilibrium". A domain $G>C$ in which the population is pure hawk at the fast equilibrium. Figure 2 shows the same graph of Figure 1, i.e. total population size with respect to $G$, but for fixed and different costs. It shows that when increasing $G$, there is at first a maximum for smaller $G$ corresponding to the mixed hawk-bully case $(G<C)$, then a minimum occurs when $G=C$ and finally the graph is monotone increasing with $G$ in the domain of a pure hawk population, $(G>C)$. In the domain $(G<C)$, there is a kind of bump (Figure 1) with a curved line corresponding to maximum population size (Figure 3). In other words, for each fixed cost $C$, there is a maximum gain. Each point along this curved line of maxima with coordinates $(G, C)$ corresponds to a given proportion of hawks which is simply given by $x_{H}^{*}=G / C$. Figure 4 shows the graph of the $x_{H}^{*}$ with respect to the cost which is a monotone decreasing function of $C$. For mixed hawk and bully populations, the maximum population size is obtained when the proportion of aggressive individuals is smaller for larger cost. In other words, the model predicts that in non aggressive populations, (small $C$ ), the proportion of hawks is expected to be larger than in aggressive populations (large $C$ ). When the cost is high, according to this model, we expect a population mainly bully with few hawks. On the contrary, when the cost is small, we expect a larger proportion of hawks in the population but with small costs, corresponding to less injuries leading to smaller mortality. In the domain $(G>C)$, the graph of population size is monotone increasing with $G$ for any cost-values. This result can be understood because 
for a fixed cost, an increase of $G$ corresponds to a richer environment with more resource available, leading as a consequence to an increase of the population size. Moreover, the maximum population sizes for the larger $G$-value ( $G=5$ on Figure 2) is smaller for larger costs. This can be understood as large cost implies a higher mortality and in turn a smaller population size.

Nevertheless, as we know, the immature needs a certain time to develop to mature stage, the model incorporating time delay may be more reasonable and worth further study, and we leave this for future study.

\section{Acknowledgements}

The work of the first author (A. Moussaoui) is supported by the National Research Project: CNEPRU B02020140100. Most part of this work was done during the stay of the first author at Applied Mathematics Laboratory of Le Havre University and granted by CNRS-France. We are very grateful to the editor and the anonymous referees for their careful reading, valuable comments and helpful suggestions, which helped us to improve the presentation of this work significantly.

\section{References}

[1] C. Accolla, D. Nérini, O. Maury, J.C. Poggiale. Analysis of functional response in presence of schooling phenomena: An IBM approach. Prog. Oceanogr., 134 (2015), 232-243.

[2] H. Amann. Ordinary Differential Equations: An Introduction to Nonlinear Analysis, Walter de Gruyter, Berlin, 1990.

[3] P. Amarasekare. Interactions between Local Dynamics and Dispersal : Insights from Single Species Models. Theor. Popul. Biol., 53 (1998), 44-59.

[4] P. Amarasekare. Spatial dynamics in a host-multi-parasitoid community. J. Anim. Ecol., 69 (2000a), $201-213$.

[5] P. Amarasekare. Coexistence of competing parasitoids on a patchily distributed host: local vs. spatial mechanisms. Ecology, 81 (2000b), 1286-1296.

[6] P. Amarasekare. The role of density-dependent dispersal in source-sink dynamics. J. Theor. Biol., 226 (2004a), $159-68$.

[7] P. Amarasekare. Spatial variation and density-dependent dispersal in competitive coexistence. P. Roy. Soc. Lond. B. Bio., 271 (2004b), 1497-1506.

[8] P. Auger, P. Roussarie. Complex ecological models with simple dynamics: from individuals to populations. Acta Biotheor., 42 (1994), 111-136.

[9] P. Auger, J.C. Poggiale. Emerging properties in population dynamics with different time scales. J. Biol. Syst., 3 (1995), 591-602.

[10] P. Auger, J.C. Poggiale. Emerging of population growth models: fast migration and slow growth. J. Theor. Biol., 182 (1996), 99-108.

[11] P. Auger, D. Pontier. Fast game theory coupled to slow population dynamics: the case of domestic cat populations. Math. Biosci., 148 (1998), 65-82.

[12] P. Auger, R. Bravo De La Parra, S. Morand. E. Sanchez. A predator-prey model with predators using hawk and dove tactics. Math. Biosci., 178 (2002), 185-200, 2002.

[13] P. Auger, B. Kooi, R. Bravo De La Parra, J. C. Poggiale. Bifurcation Analysis of a Predator-prey Model with Predators using Hawk and Dove Tactics. J. Theor. Biol., 238 (2006), 597-607.

[14] P. Auger, R. Bravo de la Parra, J.C. Poggicale, E. Sanchez, T. Nguyen-Huu. Aggregation of Variables and Applications to Population Dynamics, in Magal P, Ruan S (eds.), Structured Population Models in Biology and Epidemiology, Lecture Notes in Mathematics, Vol 1936, Mathematical Biosciences Subseries, Springer, Berlin, 209-263. 2008.

[15] P. Auger, R. Bravo De La Parra, E. Sanchez. Behavioral dynamics of two interacting hawk dove populations. Math. Mod. Meth. Appl. S., 11 (2001), 645-661.

[16] D.E. Bowler, G.T. Benton. Causes and consequences of animal dispersal strategies: relating individual behaviour to spatial dynamics. Biol. Rev., 80 (2005), 205-225.

[17] E. Chambon-Dubreuil, P. Auger, J. M. Gaillard, M. Khaladi. Effect of aggressive behaviour on age-structured population dynamics. Ecol. Model., 193 (2006), 777-786.

[18] L. Edelstein-kheset. Mathematics Models in Biology, Rondom House, New York, 1988.

[19] A. Elabdalaoui, P. Auger, R. Bravo De La Parra, B. Kooi, R. Mchich. Effects of density-dependent migrations on stability of a two-patch predator-prey model. Math. Biosci., 210 (2007), 335-354,

[20] I. Hanski. Individual behavior, population dynamics and community structure of Aphodius (Scarabaeidae) in Europe. Acta. Oecol-Oec. Gen., 7 (1986), 171-187.

[21] C. Hauert, M. Holmes, M. Doebeli. Evolutionary games and population dynamics: maintenance of cooperation in public goods games. P. Roy. Soc. Lond. B. Bio., 273 (2006), 2565-2570.

[22] J. Hofbauer, K. Sigmund. Evolutionary Games and Population Dynamics. Cambridge University Press. Cambridge. 1998.

[23] C. Lett, V. Mirabet. Modelling the dynamics of animal groups in motion. S. Afr. J. Sci., 104 (2008), $192-198$. 
[24] O. Maury, J.C. Poggiale. From individuals to populations to communities: A dynamic energy budget model of marine ecosystem size-spectrum including life history diversity. J. Theor. Biol., 324 (2013), 52-71.

[25] M. Marvá, A. Moussaoui, R. Bravo de la Parra, P. Auger. density dependent model describing age-structured population dynamics using hawk-dove tactics. J. Differ. Equ. Appl., 19 (2013), 1-13.

[26] J. Maynard Smith, G.R. Price. The logic of animal conflict. Nature, 246 (1973), 15-18.

[27] J. Maynard-Smith. The theory of games and the evolution of animal conflicts. J. Theor. Biol., 47 (1974), $209-221$.

[28] J. Maynard Smith. Evolution and the Theory of Games. Cambridge University Press. Cambridge, UK, 1982.

[29] R. Mchich, P. Auger, C. Lett. Effects of aggregative and solitary individual behaviors on the dynamics of predator-prey game models. Ecol. Model., 197 (2006), 281-289.

[30] V. Mirabet, P. Auger, C. Lett. Spatial structures in simulations of animal grouping. Ecol. Model., 201 (2007), 468-476.

[31] A. Moussaoui, P. Auger, B. Roche. Effect of hawk-dove game on the dynamics of two competing species. Acta Biotheor., $62(2014), 385-404$

[32] A. Moussaoui, P. Auger, N. Ngoc Doanh. A stage structured population model with mature individuals using hawk and dove tactics. ARIMA, 20 (2015), 127-143.

[33] J.D. Murray. Mathematical Biology, Springer-Verlag, 1989.

[34] T. Nguyen Huu, P. Auger, C. Lett, M. Márva. Emergence of global behaviour in a host-parasitoid model with densitydependent dispersal in a chain of patches. Ecol. Complex., 5 (2008), 9-21.

[35] A. Okubo, S.A. Levin. Diffusion and ecological problems: Modern perspectives. Springer Verlag, 2002.

[36] D. Pontier, P. Auger, R. Bravo de la Parra, E. Sanchez. The impact of behavioral plasticity at individual level on domestic cat population dynamics. Ecol. Model., 133 (2000), 117-124.

[37] E. Sánchez. P. Auger, R. Bravo De La Parra. Influence of Individual aggressiveness on the dynamics of competitive populations. Acta Biotheor., 45 (1997), 321-333.

[38] K. Sigmund. A survey of replicator equations, in Complexity, Language, and Life : Mathematical Approaches, eds. J. L. Casti and A. Karlqvist, Biomathematics Texts. 16, Springer, 1986.

[39] D.J.T. Sumpter, D.S. Broomhead. Relating individual behaviour to population dynamics. P. Roy. Soc. Lond. B. Bio., 268 (2001), 925-932.

[40] W.J. Sutherland. From individual behavior to population ecology. Oxford University Press. 1997.

[41] E.C. Zeeman. Dynamics of the evolution of animal conflicts. J. Theor. Biol., 89 (1981), 249-270. 